\title{
Über Umsetzungen von Harnstoff mit Alkoholen ${ }^{1}$
}

\begin{abstract}
।
\author{
Von Alfred Max Paquin
}

(Z. Naturforschg. 1, 518-523 [1946]; eingegangen am 16. April 1946)

Aus dem wissenschaftlichén Laboratorium der I.G. Farbenindustrie A.G., Höchst a. M.

Die Umsetzung von Harnstoff mit Alkoholen zu Urethanen findet in vielen Fällen, besonders bei mehrwertigen Alkoholen mit benachbarten Hydroxylgruppen, nicht statt. Dafür entstehen Derivate der Iminodicarbonsäure oder der Anhydro-(oxyalkyl-carbamid- säuren). Es wurden Katalysatoren gefunden, welche die Urethanbildung begünstigen, und solche, welche die Umsetzung nach der anderen Richtung lenken. Bei 3- und mehrwertigen Alkoholen mit benachbarten Hydroxylgruppen entstehen höhermolekulare, wasserlösliche Harze. Mehrwertige Alkohole mit nicht benachbarten Hydroxylgruppen gestatten Urethangewinnung. Spaltprodukte des 1.3-Butylen-diurethans: flüchtige Basen, Anhydro-\{[4-oxy-butyl-(2)] - carbamidsäure \}und $N . N^{\prime}-[\alpha$-Methyl-trimethylen] - harnstoff. Verwendung als Weichmacher, Duftstoffe, kautschukartige Massen, Stabilisierungsmittel für Nitrobronzelacke und Zaponlacke, Futtermittel und als Textildruckerei-Hilfsmittel.
\end{abstract}

$\mathrm{I}_{\mathrm{s}}^{\mathrm{n}}$ Verfolg der bekannten Umsetzung von Harnstoff mit Alkoholen zu Urethanen wurde gefunden, daß in vielen Fällen nur eine sehr schlechte Ausbeute, in anderen Fällen überhaupt kein Urethan zu erhalten ist. Dafür entstehen andersartige, z. Tl. schwer oder gar nicht kristallisierende Umsetzungsprodukte, die in manchen Fällen höhermolekular sind ${ }^{2,3}$.

Die Umsetzung von Harnstoff mit einwertigen Alkoholen liefert stets Urethan. Die Ausbeute hängt sehr von den Umsetzungsbedingungen ab. Durch gewisse Katalysatoren, vornehmlich durch Schwermetallsalze schwacher organischer Säuren, konnte sie bis auf $90 \%$ der Theorie gesteigert werden. Andererseits ließ sich die Bildung der Urethane zugunsten der andersartigen Produkte durch Zusatz von starken Mineralsäuren oder von Salzen dieser Säuren praktisch gänzlich unterbinden. Die Umsetzung erfolgt normalerweise bei 140 bis $200^{\circ}$. Durch erhöhten Zusatz von starken Mineralsäuren war es möglich, die gleichen Umsetzungen schon bei 50 bis $70^{\circ} \mathrm{zu}$ erzielen, so daß die Kondensation auch mit unter $100^{\circ}$ siedenden Alkoholen durchführbar war ${ }^{4}$.

1 I. Mitteilung über eine aus äußeren Gründen plötzlich abgebrochene Arbeit „Neue Verbindungen des Harnstoffs und des Sulfamids“. Weitere Mitteilungen über die bisher erzielten Ergebnisse sollen an anderer Stelle erscheinen. Die Veröffentlichung erfolgt, weil keine Möglichkeit besteht, die Arbeit fortzusetzen. Da ein Teil der Protokolle und Journale nicht mehr zur Verfügung steht, muß teils ganz auf die Wiedergabe einiger Ergebnisse, teils auf die Angabe einiger Daten und Analysen verzichtet werden. Die vom Verf. genommenen Patente und Patentanmeldungen sind als Fußnoten angegeben.
Diese Umsetzung findet aber nicht nur mit echten Alkoholen, sondern auch mit solchen Substanzen statt, die eine oder mehrere HydroxyIgruppen enthalten, z. B. Milchsäureestern, Glycerinmono- und -diestern und dergleichen. Um die störende Bildung von Allophansäureestern zu verhindern, wurde im allgemeinen bei der Umsetzung statt $1 \mathrm{Mol}$ Alkohol die 2-3-fache Menge angewandt.

Es wurde gefunden, daß es sich bei den Substanzen, die bei der Umsetzung von Harnstoff mit einwertigen Alkoholen dann entstehen, wenn eine Urethanbildung nicht stattfindet, um Derivate der Iminodicarbonsäure handelt. So konnten ohne Schwierigkeiten mehrere Iminodicarbonsäure-dialkylester hergestellt werden, von denen bereits der Iminodicarbonsäure-diäthylester ${ }^{5}$, allerdings auf andere Weise, hergestellt worden ist. Die Bildung dieser Verbindungen ist durch Kondensation zweier Urethanmoleküle miteinander zu erklären'

$2 \mathrm{RO} \cdot \mathrm{CO} \cdot \mathrm{NH}_{2}=\mathrm{RO} \cdot \mathrm{CO} \cdot \mathrm{NH} \cdot \mathrm{CO} \cdot \mathrm{OR}+\mathrm{NH}_{3}$

Zweiwertige Alkohole mit benachbarten Hydroxylgruppen setzen sich mit Harnstoff in anderer Weise um, wobei ohne Katalysatoren die Ausbeute an Urethanen schlecht ist. Mit Hilfe

2 Scheiber, Chemie u. Technologie d. künstl. Harze, S. 389 ff.

3 D.R.P. 506963

4 D.R.P. 508795 , E.P. 280238 , F.P. 706044, A.P. 1924253 .

5 B e i l s t e i n, IV. Aufl., Bd. 3, S. 27, I. Erg.-Werk Bd. 3, S. 13.

6 D.R.P. 506963, E.P. 278390, F.P. 641770, A.P. 1902889 . 
der genannten Katalysatoren läßt sich die Ausbeute jedoch beträchtlich steigern. Durch Zusatz saurer mineralsaurer Salze oder von freien Mineralsäuren wird die Urethanbildung völlig unterbunden und es entstehen Verbindungen vom Typ der Anhydro - (oxyalkyl - carbamidsäuren). Von dieser Klasse ist die Anhydro-[( $\beta$-oxyäthyl)-carbamidsäure], Oxazolidon-(2) (I) ${ }^{7}$ früher durch Umsetzung von Oxyäthylamin mit Kaliumcyanat erhalten worden. Diese Verbindungen entstehen entweder durch Umsetzung äquimolekularer oder der doppelten Mol-Mengen von Harnstoff mit zweiwertigen Alkoholen. Die Bildung ist sowohl aus dem Monourethan als auch aus dem Diurethan verständlich. Beispielsweise stellt sich die Umsetzung, ausgehend vom Glykol, folgendermaßen dar:

$$
\begin{aligned}
& \underset{\mathrm{CH}_{2} \cdot \mathrm{OH}}{\mathrm{CH}_{2} \cdot \mathrm{O} \cdot \mathrm{CO} \cdot \mathrm{NH}_{2}}=\underset{\mathrm{CH}_{2} \cdot \mathrm{NH}^{\prime}}{\mathrm{CH}_{2}} \cdot \mathrm{O}-\mathrm{CO}+\mathrm{H}_{2} \mathrm{O} \\
& \text { und I } \\
& \underset{\mathrm{CH}_{2} \cdot 0 \cdot \mathrm{CO} \cdot \mathrm{NH}_{2}}{\mathrm{CH}_{2} \cdot 0 \cdot \mathrm{CO} \cdot \mathrm{NH}_{2}}=\underset{\mathrm{CH}_{2}}{\stackrel{\mathrm{CH}}{\mathrm{CH}_{2}} \cdot \mathrm{O}} \underset{\mathrm{NH}}{\mathbf{l}} \mathrm{CO}+\mathrm{CO}_{2}+\mathrm{NH}_{3} \cdot
\end{aligned}
$$

Auch Aminoäthanol reagiert in analoger Weise:

$$
\begin{aligned}
& \mathrm{CH}_{2} \cdot \mathrm{OH}+\underset{\mathrm{CO}}{\mathrm{NH}_{2}}=\mathrm{CH}_{2} \cdot \mathrm{O} \cdot \mathrm{CO} \cdot \mathrm{NH}_{2} \\
& \stackrel{\mathrm{CH}}{2}_{\mathrm{NH}_{2}}+\underset{\mathrm{NH}_{2}}{\mathrm{CO}}=\underset{\mathrm{CH}_{2}}{\mathrm{NH}_{2}} \\
& =\begin{array}{l}
\mathrm{CH}_{2} \cdot \mathrm{O} \\
\mathrm{CH}_{2} \cdot \mathrm{NH}^{\prime}
\end{array} \mathrm{CO}+2 \mathrm{NH}_{3} .
\end{aligned}
$$

Die nach diesen Verfahren leicht zugänglich gewordene Verb. I wurde für die verschiedenartigsten Reaktionen herangezogen; u. a. gelang es, sie mit primären Aminen zu Harnstoffderivaten umzusetzen:

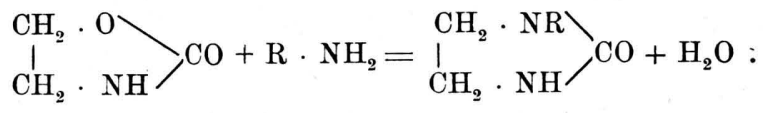

Dreiwertige Alkohole mit benachbarten Hydroxylgruppen liefern in keinem Falle bei der Umsetzung mit Harnstoff Urethane, auch nicht bei Verwendung der die Urethanbildung begünstigenden Salze. Glycerin ergibt, wenn es mit 1 oder 2 Mol. Harnstoff umgesetzt wird, identische Produkte, ein Öl, das vermutlich $\beta$-Anhydro- $\left[\right.$ ( $\alpha^{-}$ oxymethyl- $\beta$-oxy-äthyl)-carbamidsäure ], 4-Oxymethyl-[oxazolidon-(2)] (II) darstellt und in analoger Weise wie beim Glykol entstanden sein dürfte. Mit $3 \mathrm{Mol}$. Harnstoff umgesetzt, ergibt Glycerin ein hochmolekulares Harz von der vermutlichen Struktur III.

$$
\begin{aligned}
& \mathrm{CH}_{2} \cdot \mathrm{O} \\
& \stackrel{\mathrm{CH}}{\mathrm{CH}} \cdot \mathrm{NH} \\
& \stackrel{\mathrm{CH}}{\mathrm{CH}_{2} \mathrm{OH}}
\end{aligned}
$$<smiles>CC(C)CNC(C)CC(C)NC(C)COC(N)=O</smiles>

Naturgemäß könnte in Formel III die doppelte -NH-Brücke ebensogut auch in der 1.3-Stellung, und die einfache -NH-Brücke in der 2-Stellung sein, jedoch ist das für die Erkénntnis der prinzipiellen Struktur belanglos. Durch Bestimmung der abgespaltenen Mengen von Ammoniak und Kohlendioxyd sowie der Ausbeute konnte die Formel gestützt werden.

Mehrwertige Alkohole, bei denen die Hydroxylgruppen nicht an benachbarten C-Atomen sitzen, setzen sich mit Harnstoff schwerer um; die Urethane sind ohne Verwendung von Katalysatoren in mäßiger Ausbeute erhältlich ${ }^{8}$. Nach Zusatz der beschriebenen Katalysatoren wird die Ausbeute ausgezeichnet.

1.3-Butylen-monourethan, eine glycerinähnliche

7 B eilstein, IV. Aufl., Bd. 27, S. 135; K n o r r, $\mathrm{R}$ ö ß l e r, Ber. dtsch. chem. Ges. 36, 1281 [1903].

${ }^{8}$ D.R.P. $501852 .{ }^{9}$ D.R.P. 549 194. ${ }^{10}$ D.R.P. 713467.
Flüssigkeit, zeigt bei der Destillation, auch im Vakuum, Neigung, sich in geringem Maße zu zersetzen, unter Regenerierung von 1.3-Butylen-glykol und Abscheidung unlöslicher Cyanursäure, welche milchige Trübungen hervorruft. 1.3-Butylen-diurethan zeigt eine interessante thermische Zersetzung ${ }^{10}$ : neben einer geringen Menge piperidinartig riechender Aminbasen erhält man als Hauptausbeute ein Gemisch von Anhydro-\{[4oxy-butyl-(2)]-carbamidsäure \}, 4-Methyl-2-oxo-

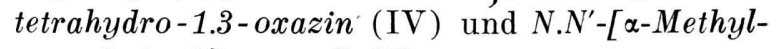
trimethylen]-harnstoff (V).

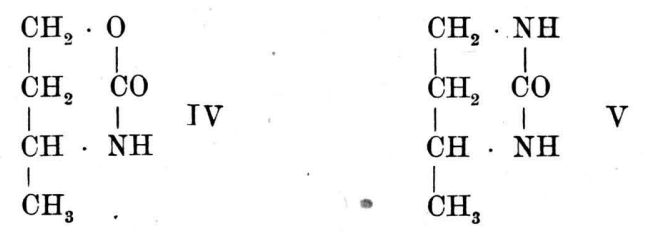


Diese beiden Verbindungen lassen sich leicht voneinander trennen. Die Bildung der Verb. $\mathrm{V}$ ist unter Abspaltung von Wasser und Kohlendioxyd unter Umlagerung erfolgt. Die Umsetzung von 2 Mol. Harnstoff mit 1 Mol. Butylenglykol ohne Zugabe von Katalysatoren, welche zu einer mäßigen Ausbeute von Diurethan führt, ist stets mit Kohlendioxydabspaltung verbunden. Es konnte nachgewiesen werden, daß dabei Verb. IV aus primär gebildetem Diurethan entsteht. Bei Gegenwart von Schwermetallsalzen organischer Säuren tritt keine Kohlendioxydabspaltung ein (Verb. IV entsteht nicht mehr) und die Urethanausbeute erhöht sich außerordentlich.

\section{Spezielle Verwendungen für die Umsetzungsprodukte}

Die meisten Umsetzungsprodukte bieten sich als Weichmacher oder als Zusatzprodukte zu Kunststoffen aller Art an ${ }^{11}$. Sie haben z. Tl. recht gute Eigenschaften. In folgendem werden solche Umsetzungsprodukte angeführt, die infolge ihrer eigenartigen Eigenschaften entweder schon von besonderer Bedeutung sind oder eine solche noch erlangen können.

a) Riechstoffe. Es wurde beobachtet, daß einige höhere Urethane einen angenehmen, teerosenartigen Duft aufweisen ${ }^{12}$. An Stärke und Schönheit nimmt der Duft zu, wenn für die Urethanbildung höhere aliphatische Alkohole oder. solche mit verzweigter Seitenkette angewandt werden ${ }^{13}$. Weitere Nuancierungen und Verbesserungen konnten durch Substitution von einem oder beiden Wasserstoffatomen in der Aminogruppe erzielt werden ${ }^{14}$.

b) Kautschukartige Kunststoffe. Umsetzungsprodukte von Harnstoff mit hochpolymeren Alkoholen ergeben kautschukartige, sehr elastische Massen ${ }^{15}$, welche ihre Elastizität noch nach Jahren unverändert erhalten. Die Löslichkeitseigenschaften und andere physikalische Daten lassen sich weitgehend durch die Umsetzungsbedingungen und durch die Verwendung von speziellen Harnstofftypen oder -substitutionsprodukten modifizieren. Teils für sich allein, teils in Kombination mit anderen Kunststoffen scheinen diese Produkte dazu berufen zu sein, in der Kunststoffindustrie eine Rolle zu spielen.

c) Herstellung haltbarer Nitrolacke, die kupferhaltige Bronzen enthalten, und solcher Zaponlacke, welche polierte kupferhaltige Metalle nicht bräunen. Das Gelatinieren und Vergrünen von Nitrolacken, die kupferhaltige Metallbronzen enthalten, welche zum Bronzieren weite Verbreitung gefunden haben, ebenso wie die Herstellung solcher Zaponlacke, welche blanke Oberflächen auf kupferhaltigen Metall-Legierungen nicht dunkel färben, wird durch einen kleinen Zusatz gewisser Umsetzungsprodukte von Harnstoff mit Alkoholen vermieden bzw. erreicht. In der Lackindustrie hat das Verfahren Bedeutung erlangt ${ }^{16}$.

${ }^{11}$ D.R.P. $549194 .{ }^{12}$ D.R.P. ang. ${ }^{13}$ D.R.P. ang. d) Harnstoffumsetzungsprodukte mit mehrwertigen höheren Alkoholen oder Kohlehydraten als Futtermittel. Umsetzungsprodukte von Harnstoff mit höheren mehrwertigen Alkoholen, z. B. Pentaerythrit, Sorbit, Mannit u. ähnl., sowie solche mit Stärke oder stärkehaltigen Substanzen (Kartoffeln) haben angenehmen Geruch und Geschmack und werden bei Dauerfütterungsversuchen von Haustieren ohne Schaden vertragen. Die Produkte haben gegenüber dem Ausgangsmaterial einen erheblich gesteigerten Nährwert und können - auch bei Nicht-Wiederkäuern - eiweißreiche Futtermittel entweder ganz oder teilweise ersparen. Nebenprodukt ist das wertvolle Düngemittel Ammonsulfat. Das Verfahren hat in Notzeiten zur Einsparung eiweißreicher Auslandsfuttermittel erhebliche Bedeutung ${ }^{17}$.

e) Textildruckerei-Hilfsmittel. Eine Reihe von Umsetzungsprodukten von Harnstoffen mit Alkoholen hat sich als ausgezeichneter Zusatz zu Druckereipasten erwiesen. Unter Verwendung der üblichen Druckund Fixiermittel erhält man besonders kräftige und farbreine Drucke, die sich durch Gleichmäßigkeit auszeichnen. Auch im Arbeitsgang selbst werden Vorteile erzielt ${ }^{18}$.

\section{Beschreibung der Versuche}

1-6. Benzylurethan.

\begin{tabular}{|c|c|c|c|c|c|}
\hline 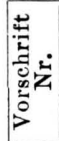 & $\begin{array}{c}\text { Harn- } \\
\text { stoff } \\
\text { Gew.- } \\
\text { Tle. }\end{array}$ & $\begin{array}{c}\text { Benzyl- } \\
\text { alkohol } \\
\text { Gew.- } \\
\text { Tle. }\end{array}$ & $\begin{array}{c}\text { Zusätze } \\
\text { Gew.-Tle. }\end{array}$ & $\begin{array}{l}\text { Bedin- } \\
\text { gungen }\end{array}$ & $\begin{array}{l}\text { Urethan } \\
\text { Ausb.in } \\
\% \text { d.Th. }\end{array}$ \\
\hline 1 & 60 & 324 & - & $\begin{array}{l}\text { 5 Stunden } \\
175-185^{\circ}\end{array}$ & 56 \\
\hline 2 & 60 & 216 & $\begin{array}{r}4 \text { Zink- } \\
\text { acetat }\end{array}$ & $\begin{array}{l}8 \text { Stunden } \\
150-160^{\circ}\end{array}$ & 87 \\
\hline 3 & 60 & 216 & $\begin{array}{l}4 \text { Stanni- } \\
\text { chlorid }\end{array}$ & $\begin{array}{l}8 \text { Stunden } \\
150-160^{\circ}\end{array}$ & 92 \\
\hline 4 & 60 & 216 & 1 konz. & $\begin{array}{l}5 \text { Stunden } \\
175-185^{\circ}\end{array}$ & $\begin{array}{l}\text { kein } \\
\text { Urethan* }\end{array}$ \\
\hline 5 & 60 & 216 & $\underset{\text { bisulfat }}{6 \text { Ammon- }}$ & $\begin{array}{l}5 \text { Stunden } \\
175-185^{\circ}\end{array}$ & $\underset{\text { Eein }}{\text { Urethan* }}$ \\
\hline 6 & 60 & 216 & 8 Glycerin & $\begin{array}{c}5 \text { Stunden } \\
175-185^{\circ}\end{array}$ & $\begin{array}{l}\text { kein } \\
\text { Urethan* }\end{array}$ \\
\hline
\end{tabular}

* Weichharz, analysenrein kristallin.

7. Iminodicarbonsäure-dibenzylester. Die nach den Vorschriften 4, 5 und 6 erhaltenen Weichharze werden in gutem Vakuum fraktioniert destilliert. Die analysenreine Substanz erstarrt kristallin. Die Analyse stimmt auf eine Verbindung der Formel $\mathrm{C}_{16} \mathrm{H}_{15} \mathrm{O}_{4} \mathrm{~N}$; demnach ist durch Kondensation zweier Moleküle Benzylurethan Iminodicarbonsäure-dibenzylester entstanden. Die Ausbeute ist gut. (Unterlagen stehen nicht zur Verfügung.)

${ }^{14}$ D.R.P. ang. ${ }^{15}$ D.R.P. $519049 .{ }^{16}$ D.R.P. 529990.

${ }^{17}$ D.R.P. ang. ${ }^{18}$ D.R.P. 543462. 
8-12. Isobutylurethan.

\begin{tabular}{|c|c|c|c|c|c|}
\hline 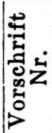 & \begin{tabular}{|c} 
Harn- \\
stoff \\
Gew.- \\
Tle.
\end{tabular} & \begin{tabular}{|c} 
Isobu- \\
tanol \\
Gew.- \\
Tle.
\end{tabular} & $\begin{array}{c}\text { Zusätze } \\
\text { Gew.-Tle. }\end{array}$ & $\begin{array}{l}\text { Bedin- } \\
\text { gungen }\end{array}$ & $\begin{array}{l}\text { Urethan } \\
\text { Ausb.in } \\
\% \text { d. Th. }\end{array}$ \\
\hline 8 & 60 & 222 & - & $\begin{array}{c}150 \text { Stunden } \\
108-126^{\circ}\end{array}$ & 49 \\
\hline 9 & 60 & 222 & $\begin{array}{l}6 \text { Blei- } \\
\text { acetat }\end{array}$ & $\begin{array}{r}75 \text { Stunden } \\
108-126^{0}\end{array}$ & 88 \\
\hline 10 & 60 & 222 & $\begin{array}{l}5 \text { Kobalt- } \\
\text { chlorid }\end{array}$ & $\begin{array}{r}75 \text { Stunden } \\
108-126^{0}\end{array}$ & 92 \\
\hline 11 & 60 & 144 & $\begin{array}{c}5 \text { 85-proz. } \\
\mathrm{H}_{3} \mathrm{PO}_{4}\end{array}$ & $\begin{array}{l}12 \text { Stunden } \\
80-85^{\circ}\end{array}$ & $\underset{\text { Urethan* }}{\text { kein }}$ \\
\hline 12 & 60 & 144 & $\begin{array}{c}\text { sehr } \\
\text { schwacher } \\
\text { HCl- } \\
\text { Strom }\end{array}$ & $\begin{array}{l}10 \text { Stunden } \\
70-75^{\circ}\end{array}$ & $\begin{array}{l}\text { kein } \\
\text { Urethan* }\end{array}$ \\
\hline
\end{tabular}

* Viscoses Öl, analysenrein, kristallin.

13. Iminodicarbonsäure-diisobutylester. Die nach 11 und 12 erhaltenen öle werden im Vakuum fraktioniert. Die reine Substanz erstarrt kristallin. Die Ausbeute ist gut. Die Analyse stimmt auf Iminodicarbonsäurediisobutylester $\mathrm{C}_{10} \mathrm{H}_{19} \mathrm{O}_{4} \mathrm{~N}$. (Unterlagen stehen nicht zur Verfügung.)

\section{Iminodicarbonsäure-diäthylester}

a, 14-15. 92 Gew.-Tle. absol. Athylalkohol (2 Mol.) und 60 Gew.-Tle. Harnstoff (1 Mol.) werden bei $40^{\circ}$ bis zur Sättigung gerührt, dann läßt man 8 Gew.-Tle. konz. Schwefelsäure eintropfen und rührt 7-8 Stdn. bei $60-65^{\circ}$. Nach Abtrennen des Ammonsulfates mittels Alkohol-Acetons erhält man ein viscoses Öl, das in reinem Zustande kristallin wird.

Das Öl wird bei $15 \mathrm{~mm}$ fraktioniert. Das zwischen $130-140^{\circ}$ Übergehende wird nochmals fraktioniert und erstarrt dann kristallin. Schmp. $50^{\circ}$. Die Ausbeute ist gut. Die Analyse stimmt auf Iminodicarbonsäurediäthylester $\mathrm{C}_{6} \mathrm{H}_{11} \mathrm{O}_{4} \mathrm{~N}$.

b, 16. 267 Gew.-Tle. Carbaminsäureäthylester, 15 Gew.Tle. Glycerin und 7 Gew.-Tle. 85-proz. Phosphorsäure werden am Kühlrohr unter Rühren erhitzt. Bei $185^{\circ}$ beginnt lebhafte Ammoniakabspaltung. Nach 4 Stdn. wird eine Siedetemperatur von $220^{\circ}$ erreicht. Weiteres Rühren bei $215-220^{\circ}$ während 6 Stdn., dann wird wie bei a gereinigt und fraktioniert. Man erhält reinen Iminodicarbonsäure-diäthylester in guter Ausbeute.

17-18. Iminodicarbonsäure - bis - (milchsäure - butyl ester)-ester, $\mathrm{C}_{4} \mathrm{H}_{9} \cdot \mathrm{O} \cdot \mathrm{CO} \cdot \mathrm{CH}\left(\mathrm{CH}_{3}\right) \cdot \mathrm{O} \cdot \mathrm{CO} \cdot \mathrm{NH} \cdot \mathrm{CO}$ - $\mathrm{O} \cdot \mathrm{CH}\left(\mathrm{CH}_{3}\right) \cdot \mathrm{CO} \cdot \mathrm{O} \cdot \mathrm{C}_{4} \mathrm{H}_{\mathbf{8}} .438 \mathrm{Gew}$.-Tle. Milchsäurebutylester (3 Mol.), 60 Gew.-Tle. Harnstoff (1 Mol.) und 8 Gew.-Tle. pulv. Kaliumbisulfat werden am Rückflußkühler unter Rühren 4 Stdn. im Sieden gehalten. Die Temperatur steigt hierbei auf über $200^{\circ}$. Nach dem Reinigen erhält man ein viscoses Öl, das durch mehrfaches Fraktionieren im Vakuum gereinigt wird. Die Analyse stimmt auf $\mathrm{C}_{16} \mathrm{H}_{27} \mathrm{O}_{8} \mathrm{~N}$. (Unterlagen stehen nicht zur Verfügung.)
19-20. Iminodicarbonsäure - bis - (glycerindiacetylester)-ester, $\left(\mathrm{CH}_{3} \cdot \mathrm{CO} \cdot \mathrm{O} \cdot \mathrm{CH}_{2}\right)_{2} \mathrm{CH} \cdot \mathrm{O} \cdot \mathrm{CO} \cdot \mathrm{NH}$ - $\mathrm{CO} \cdot \mathrm{O} \cdot \mathrm{CH}\left(\mathrm{CH}_{2} \cdot \mathrm{O} \cdot \mathrm{CO} \cdot \mathrm{CH}_{3}\right)_{2} \cdot 176$ Gew.-Tle. Diacetin (1 Mol.), 60 Gew.-Tle. Harnstoff (1 Mol.) und 6 Gew.-Tle. 85-proz. Phosphorsäure werden 8 Stdn. am Kühlrohr bei $165-170^{\circ}$ gerührt. Nach dem Reinigen erhält man ein dickflüssiges öl, das durch mehrfaches Fraktionieren im Vakuum gereinigt wurde. Die Analyse stimmt auf $\mathrm{C}_{16} \mathrm{H}_{23} \mathrm{O}_{12} \mathrm{~N}$. (Unterlagen stehen nicht zur Verfügung.)

21-24. Methylcyclohexyl-urethan.

\begin{tabular}{|c|c|c|c|c|c|}
\hline 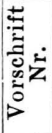 & \begin{tabular}{|c} 
Harn- \\
stoff \\
Gew.- \\
Tle.
\end{tabular} & $\begin{array}{c}\text { Methyl- } \\
\text { cyclo- } \\
\text { hexanol } \\
\text { Gew.- } \\
\text { Tle. }\end{array}$ & $\begin{array}{c}\text { Zusätze } \\
\text { Gew.-Tle. }\end{array}$ & $\begin{array}{l}\text { Bedin- } \\
\text { gungen }\end{array}$ & $\begin{array}{l}\text { Urethan } \\
\text { Ausb.in } \\
\% \text { d.Th. }\end{array}$ \\
\hline 21 & 60 & 345 & - & 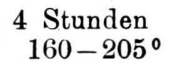 & 18 \\
\hline 22 & 60 & $34 \check{5}$ & - & $\begin{array}{l}6 \text { Stunden } \\
150-160^{\circ}\end{array}$ & 56 \\
\hline 23 & 60 & 345 & $\begin{array}{l}7 \text { Zink- } \\
\text { acetat }\end{array}$ & $\begin{array}{l}5 \text { Stunden } \\
150-155^{\circ}\end{array}$ & 83 \\
\hline 24 & 60 & 345 & $\begin{array}{c}785 \text {-proz. } \\
\mathrm{H}_{3} \mathrm{PO}_{4}\end{array}$ & $\begin{array}{l}5 \text { Stunden } \\
160-185^{\circ}\end{array}$ & $\begin{array}{l}\text { kein } \\
\text { Urethan* }\end{array}$ \\
\hline
\end{tabular}

* Glasiges Weichharz.

25. Iminodicarbonsäure-bis-[methylcyclohexylester]. Das nach 24 erhaltene Weichharz wird durch mehrfaches Fraktionieren im Vakuum gereinigt. Die reine Substanz bleibt selbst nach jahrelangem Aufbewahren weichharzartig-amorph. Die Ausbeute ist gut. Die Analyse stimmte auf $\mathrm{C}_{16} \mathrm{H}_{29} \mathrm{O}_{4} \mathrm{~N}$. (Unterlagen stehen nicht zur Verfügung.)

\section{Glykol-diurethan und Anhydro-[( $\beta$-oxy-äthyl)-carb- amidsäure]

26. 62 Gew.-Tle. Glykol (1 Mol.) und 60 Gew.-Tle. Harnstoff (1 Mol.) werden am Rückflußkühler mit anschließendem absteigendem Kühler 2 Stdn. bei '135 bis $145^{\circ}$, dann 2 Stdn. bei $145-165^{\circ}$ und 1 Stde. bei 165 bis $175^{\circ}$ gerührt. Neben der Ammoniakabspaltung wird Wasser gebildet, welches allmählich abdestilliert. Die Umsetzung ist beendet, wenn etwa 1 Mol Wasser abgespalten worden ist. Das Umsetzungsprodukt erstarrt nach Fraktionieren im Vakuum kristallin. Schmp. 910. Die Substanz ist identisch mit Verb. I.

27. 62 Gew.-Tle. Glykol (1/Mol) und 120 Gew.-Tle. Harnstoff (2 Mol.) werden am Rückflußkühler $1^{1} / 2$ Stdn. bei $150-155^{\circ}$ gerührt. Nach dem Abkühlen erhält man einen Kristallbrei, aus dem nach dem Umkristallisieren aus Alkohol Glykol-diurethan in einer Ausbeute von $19 \%$ d. Th. erhalten wird. Der größte Teil des Umsetzungsproduktes, der sich in der Mutterlauge befindet, stellt ein Weichharz dar, das sich nach der Reinigung als Verb. I erweist.

28. 62 Gew.-Tle. Glykol (1 Mol), 120 Gew.-Tle. Harnstoff $(2 \mathrm{Mol})$ und 8 Gew.-Tle. Manganacetat werden $1^{1 / 2} \operatorname{Stdn}$. bei $135-145^{\circ}$ und $1^{1} / 2$ Stdn. bei $145-155^{\circ}$ ge- 
rührt. Nach dem Abkühlen erhält man einen steifen Kristallbrei, aus dem nach dem Anteigen mit AlkoholAceton eine Rohausbeute von Glykol-diurethan von $78 \%$ d. Th. erhalten wird.

29. 62 Gew.-Tle. Glykol (1 Mol.), 120 Gew.-Tle. Harnstoff (2 Mol.) und 4 Gew.-Tle. Phosphorsäure werden 2 Stdn. bei $135-145^{\circ}, 2$ Stdn. bei $145-155^{\circ}$ und 2 Stdn. bei $155-165^{\circ}$ am Rückflußkühler gerührt, wobei neben der Ammoniakabspaltung lebhafte Kohlendioxydentwicklung auftritt. Nach dem Abkühlen erhält man ein Weichharz, das keine Spur Diurethan enthält. Nach dem Reinigen durch Vakuumfraktionierung erweist sich das Umsetzungsprodukt als Verb. I.

30. 61 Gew.-Tle. Aminoäthanol (1 Mol.) und 60 Gew.Tle. Harnstoff ( $1 \mathrm{Mol}$ ) werden $2^{1 / 2} \mathrm{Stdn}$. bei $125-135^{0}$ und $2^{1} / 2$ Stdn. bei $135-150^{\circ}$ gerührt. Das amorphe Umsetzungsprodukt wird fraktioniert; es erweist sich als Verb. I.

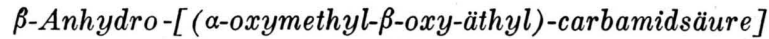

31. 92 Gew.-Tle. Glycerin (1 Mol) und 60 Gew.-Tle. Harnstoff (1 Mol) werden $5 \mathrm{Stdn}$. bei $135-145^{\circ}$ gerührt. Das viscos-ölige Umsetzungsprodukt wird im Vakuum fraktioniert. Der Durchschnitt mehrerer Analysen ergibt Werte, die mit hinreichender Genauigkeit auf die Verbindung $\mathrm{C}_{4} \mathrm{H}_{7} \mathrm{O}_{2} \mathrm{~N}$ (II) stimmen.

32. 92 Gew.-Tle. Glycerin (1 Mol) und 120 Gew.-Tle. Harnstoff (2 Mol) werden nach 31 umgesetzt. Neben Ammoniak entsteht reichlich Kohlendioxyd. Wasser wird nicht beobachtet. Die Analysenwerte stimmen mit denen von 31 überein. Die Ausbeuten entsprechen etwa der Theorie.

\section{Umsetzungsprodukt aus Glycerin mit 3 Mol Harnstoff (III)}

33-35. 92 Gew.-Tle. Glycerin und 180 Gew.-Tle. Harnstoff werden $1^{1 / 2}$ Stdn. bei $130-140^{0}$ und $2^{1 / 2}$ Stdn. bei $155-165^{\circ}$ gerührt. Die hellbraune Schmelze wird auf ein Blech gegossen. Man erhält ein hartes sprödes Harz, das leicht wasserlöslich ist und Feuchtigkeit aus der Luft anzieht. Die quantitative Bestimmung ergab: 75 Gew.-Tle. Ammoniak, 127 Gew.-Tle. Kohlendioxyd und 68 Gew.-Tle. Harz. Für die yermutlich entstandene kettenförmige Verbindung errechnet sich: Ammoniak $=17 \cdot(9 n-2) / 2 n=68-76,5$ (bei $n=2-500)$, Kohlendioxyd $=44(3 n-2) / n=88$ bis 132 (bei $n=2-500)$, Ausbeute $=(105+n \cdot 63,5) / n$ $=116-63,7$ (bei $n=2-500$ ).

An Hand der leicht aufstellbaren Tabelle kann auf Grund der Versuchsergebnisse geschlossen werden, daß die Gliederzahl $n$ der Kette eine Größenordnung von $10-50$ haben muß.

Führt man die Reaktion unter Zusatz von 8 Gew.Tln. Zinkacetat bei 4 -stdg. Rühren bei $140-145^{\circ}$ oder unter Zusatz von 4 Gew.-Tln. Phosphorsäure bei

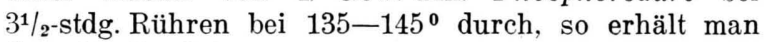
dasselbe Produkt.

\section{3-Butylen-monourethan}

36. 270 Gew.-Tle. Butylenglykol (3 Mol) und 60 Gew.Tle. Harnstoff (1 Mol) werden $2 \mathrm{Stdn}$. bei $175-185^{\circ}$ und 3 Stdn. bei $180-200^{\circ}$ gerührt. Bei der Fraktionierung bei $130-190^{\circ}(15 \mathrm{~mm})$ erhält man das Monourethan, das rein bei $174-178^{\circ}$ übergeht. Ausb. 52\% d. Th.; wird die Umsetzung unter Zugabe von Zinkacetat durchgeführt, so steigt die Ausbeute auf $88 \%$.

$$
\begin{array}{r}
\mathrm{C}_{5} \mathrm{H}_{11} \mathrm{O}_{3} \mathrm{~N} \text {. Gef. C 44,86. H 8,71, N 10,23. } \\
\text { Ber. C 45,10, H 8,38, N 10,52. }
\end{array}
$$

\section{3-Butylen-diurethan}

37. 90 Gew.-Tle. 1.3-Butylenglykol (1 Mol), 120 Gew.Tle. Harnstoff $(2 \mathrm{Mol})$ und 6 Gew.-Tle. Zinkacetat werden bei $175-185^{\circ}$ gerührt. Das kristallin erstarrende Umsetzungsprodukt wird aus Wasser umkristallisiert, in dem es heiß leicht, kalt schwer löslich ist. Schmp. $152^{\circ}$. Ausb. $78 \%$ d. Th.

\section{$\mathrm{C}_{6} \mathrm{H}_{12} \mathrm{O}_{4} \mathrm{~N}_{2}$. Gef. C 40,88, H 6,91, N 15,78. Ber. C 40,80, H 6,83, N 15,92.}

\section{Weichharz aus 1.3-Butylenglykol}

38. 90 Gew.-Tle. 1.3-Butylenglykol (1 Mol), 120 Gew.Tle. Harnstoff $(2 \mathrm{Mol})$ und 5 Gew.-Tle. 85-proz. Phosphorsäure werden $2^{1} / 2$ Stdn. bei $145-155^{0}$ und 3 Stdn. bei $160-170^{\circ}$ gerührt. Durch Bildung von Ammoncarbonat durch abgespaltenes Ammoniak und Kohlendioxyd verstopft sich der Kühler, so daß er von Zeit zu Zeit ausgewechselt werden muß. Nach Vakuumbehandlung erhält man ein helles, wasserlösliches Weichharz.

\section{Anhydro-\{[4-oxy-butyl-(2)]-carbamidsäure $\}$ (IV)}

39. 200 Gew.-Tle. 1.3-Butylen-monourethan und 10 Gew.-Tle. pulv. Chlorzink werden am Rückflußkühler mit anschließendem absteigendem Kühler só lange im Sieden gehalten, als noch Wasser übergeht. Das Umsetzungsprodukt wird bei $10-12 \mathrm{~mm}$ fraktioniert, wobei das von $185-195^{\circ}$ übergehende Destillat technisch reine Verb. IV darstellt. Aus Aceton umkristallisiert: Schmp. 910. Ausb. 117 Gew.-Tle.

\section{$\mathrm{C}_{5} \mathrm{H}_{9} \mathrm{O}_{2} \mathrm{~N}$. Gef. C 52,31, H 7,89, N 12,10. Ber. C 52,20, H 7,83, N 12,17.}

40. Aminbasen aus 1.3-Butylen-diurethan: 176 Gew.Tle. 1.3-Butylen-diurethan (1 Mol) werden unter Rühren bei absteigendem Kühler allmählich auf $240^{\circ}$ erhitzt und bei dieser Temperatur gehalten. Es destilliert fortlaufend eine gelbbraune Flüssigkeit von piperidinartigem Geruch. Die Ausbeute schwankt sehr mit der Art des Erhitzens und beträgt im Durchschnitt 46 Gew.-Tle. Uber Fraktionierung und weitere Umsetzungen der Aminbasen kann wegen Fehlens der Unterlagen nicht berichtet werden.

Die Gewinnung der Basen kann auch in der Weise erfolgen, daß von einem Ansatz zur Gewinnung von Butylendiurethan, also etwa nach Vorschrift 37, ausgegangen wird. Die Erhitzung wird hierbei entsprechend auf $240^{\circ}$ getrieben.

41. Der nach 40 hinterbleibende Destillationsrück: stand erstarrt kristallin. Er wird mit warmem Aceton angeteigt und abgesaugt. Aus der klaren Lösung kristallisiert Verb. IV. Nach erschöpfender Auslaugung 
ergeben die Acetonauszüge eine Ausbeute an technisch reinem Produkt von 70 Gew.-Teilen.

42. $N \cdot N^{\prime}-\left[\alpha-M e t h y l\right.$-trimethylen]-harnstoff ${ }^{19} \mathrm{~V}$. Der Rückstand, des Acetonauszuges von 41 wird aus Alkohol umkristallisiert. Das analysenreine Produkt schmilzt bei $201^{\circ}$ unzersetzt und siedet bei $330^{\circ}$ mit beginnender Zersetzung. Ausb. 48 Gew.-Tle. $N \cdot N^{\prime}$-[ $\alpha$-Methyl-trimethylen]-harnstoff.

$$
\begin{array}{cl}
\mathrm{C}_{5} \mathrm{H}_{10} \mathrm{ON}_{2} \text {. Gef. C 52,82, H 8,83, N 24,42. } \\
\text { Ber. C 52,65, H 8,78, N 24,58. }
\end{array}
$$

43. Kondensationsprodukt aus Polyvinylalkohol und Harnstoff. 270 Gew.-Tle. Polyvinylalkohol, 240 Gew.Tle. Harnstoff und 500 Gew.-Tle. Wasser werden bei $100^{\circ}$ gerührt. Das Sieden soll so lebhaft sein, daß stets ein kleiner Teil des Wasserdampfes aus dem Kühler entweicht. So läßt sich nach etwa 6 Stdn. die Tem-

19 Auf anderem Wege dargestellt durch J. T a f el u. R. W e in s c h e n k, Ber. dtsch. chem. Ges. 33, 3379 [1900]. peratur auf $125^{\circ}$ steigern. Die Masse wird, bei dauernder Abspaltung von Ammoniak und Kohlendioxyd, welche als Ammoncarbonat den Kühler verstopfen, immer viscoser, gummiartiger und fadenziehend. Schließlich wird der Kühler entfernt und allmählich bis auf $140^{\circ}$ erhitzt. Die Masse ist bis zuletzt noch rührbar. In kaltem Zustande ähnelt sie Kautschuk, ist äußerst elastisch und nimmt selbst bei starken Deformationen ihre ursprüngliche Form wieder an. Nach einer Lagerzeit von 12 Jahren war die Elastizität unverändert geblieben.

44. Kondensationsprodukt aus Polyvinylalkohol, Harnstoff und Glykol. 270 Gew.-Tle. Polyvinylalkohol, 460 Gew.-Tle. Harnstoff und 450 Gew.-Tle. Äthylenglykol werden 4 Stdn. auf $125-150^{\circ}$ erhitzt. Die Masse wird allmählich gummiartig, läßt sich aber bis zuletzt noch rühren. Im Vakuum wird alles Flüchtige abgetrieben und es hinterbleibt eine kautschukartige Masse ähnlich derjenigen von 43. Die Elastizität, war nach 12 Jahren noch unverändert.

\title{
Über variable Erbmerkmale der Flügeltracheen bei Ephestia und ihre Beziehungen zum Geäder
}

\author{
Von Karl Henke und Christel Berhorn \\ - Aus dem Zoologischen Institut der Universität Göttingen \\ (Z. Naturforschg. 1, 523-530 [1946]; eingegangen am 13. Juli 1946)
}

Die Gliederung des imaginalen Tracheensystems auf dem Hinterflügel von Ephestia ist von der des vorher ausgebildeten pupalen Systems relativ unabhängig und wird auch durch den Verlauf der Flügellakunen nur unvollständig bestimmt. Die Variabilität zweier seiner Hauptstämme ist bei verschiedenen Inzuchtlinien nach Art und Ausmaß verschieden. Die Entwicklung dieser Tracheenstämme richtet sich unter anderem nach dem Verhalten zweier Lakunenäste, die in Abhängigkeit von ihrem Abstand, von Außenbedingungen und einer Anzahl analysierter Gene unter der Wirkung mindestens zweier verschiedener Entwicklungsfaktoren miteinander verschmelzen können. Der Bereich dieser Lakunen wird um so häufiger von beiden Tracheen gleichzeitig besetzt, je weniger weit die Verschmelzung fortschreitet, am häufigsten wenn sie ganz ausbleibt. Im übrigen variieren die beiden Trächeenstämme in Abhängigkeit voneinander, indem sie sich in der Besetzung bestimmter Abschnitte des Geäders gegenseitig vertreten. Bei einem Inzuchtstamm mit besonders großer Variabilität der imaginalen Tracheen verschiebt sich das Verhältnis ihrer Ausbildungsgrade mit dem Ausmaß der Verschmelzung der erwähnten Aderäste. Die beobachteten Gesetzmäßigkeiten sind mit der Annahme zu deuten, daß die Ausbildung eines imaginalen Tracheenastes die Entwicklung weiterer Äste in seiner Umgebung hemmt.

$\mathrm{E}^{\prime}$ rbfaktoren mit variabler Manifestation bereiten zwar der genetischen Analyse besondere Schwierigkeiten, sind aber für die entwicklungsphysiologische Betrachtung oft um so aufschlußreicher, weil die von ihnen beeinflußten Merkmale vielfach Entwicklungskorrelationen erkennen lassen, welche einerseits die Bedingungen einzelner Organbildungen, andererseits die Wirkungsweise bestimmter Erbfaktoren beleuchten.
Manche Entwicklungsleistungen beginnen erst unter dem Einfluß bestimmter Mutationen zu variieren. Die Träger der Mutation weichen dann bald nur zum Teil, bald in wechselndem Ausmaß, schließlich auch wohl in einer zunächst mehr als qualitativ verschiedenartig erscheinenden Weise von dem durch die Wildform repräsentierten Normaltypus ab. Zu der letzten Gruppe der qualitativ variablen Mutationswirkungen sind z. B. solche Fälle zu rechnen, in denen eine $\mathrm{Mu}-$ tation in verschiedenen Körperregionen ihres Trä- 